\title{
Correction to: Mitochondrial Neuroglobin Is Necessary for Protection Induced by Conditioned Medium from Human Adipose-Derived Mesenchymal Stem Cells in Astrocytic Cells Subjected to Scratch and Metabolic Injury
}

\section{Eliana Baez-Jurado ${ }^{1}$. Gina Guio-Vega ${ }^{1}$. Oscar Hidalgo-Lanussa ${ }^{1}$. Janneth González ${ }^{1}$ - Valentina Echeverria ${ }^{2,3}$. Ghulam Md Ashraf ${ }^{4}$. Amirhossein Sahebkar ${ }^{5,6,7}$. George E. Barreto ${ }^{1}[$}

Published online: 9 December 2021

๑) Springer Science+Business Media, LLC, part of Springer Nature 2021

Correction to: Molecular Neurobiology (2019) 56:5167-5187

https://doi.org/10.1007/s12035-018-1442-9

In the original manuscript, the authors identified a duplicate image of the western blot referring to catalase (CAT) - Figure 6 (BSS5 untransfected and SCB BSS5 groups). This does not alter the results nor conclusions drawn in any way.
The authors sincerely apologize for this unintentional error.

The original article can be found online at https://doi.org/10.1007/ s12035-018-1442-9.

George E. Barreto

gsampaio@javeriana.edu.co; gesbarreto@gmail.com

1 Departamento de Nutrición y Bioquímica, Facultad de Ciencias, Pontificia Universidad Javeriana, Bogotá, D.C., Colombia

2 Facultad de Ciencias de la Salud, Universidad San Sebastian, Lientur 1457, 4080871 Concepción, Chile

3 Research \& Development Service, Bay Pines VA Healthcare System, Bay Pines, FL 33744, USA

4 King Fahd Medical Research Center, King Abdulaziz University, Jeddah 21589, Saudi Arabia

5 Neurogenic Inflammation Research Center, Mashhad University of Medical Sciences, Mashhad, Iran

6 Biotechnology Research Center, Pharmaceutical Technology Institute, Mashhad University of Medical Sciences, Mashhad, Iran

7 School of Pharmacy, Mashhad University of Medical Sciences, Mashhad, Iran 
The corrected Figure 6 is provided below.

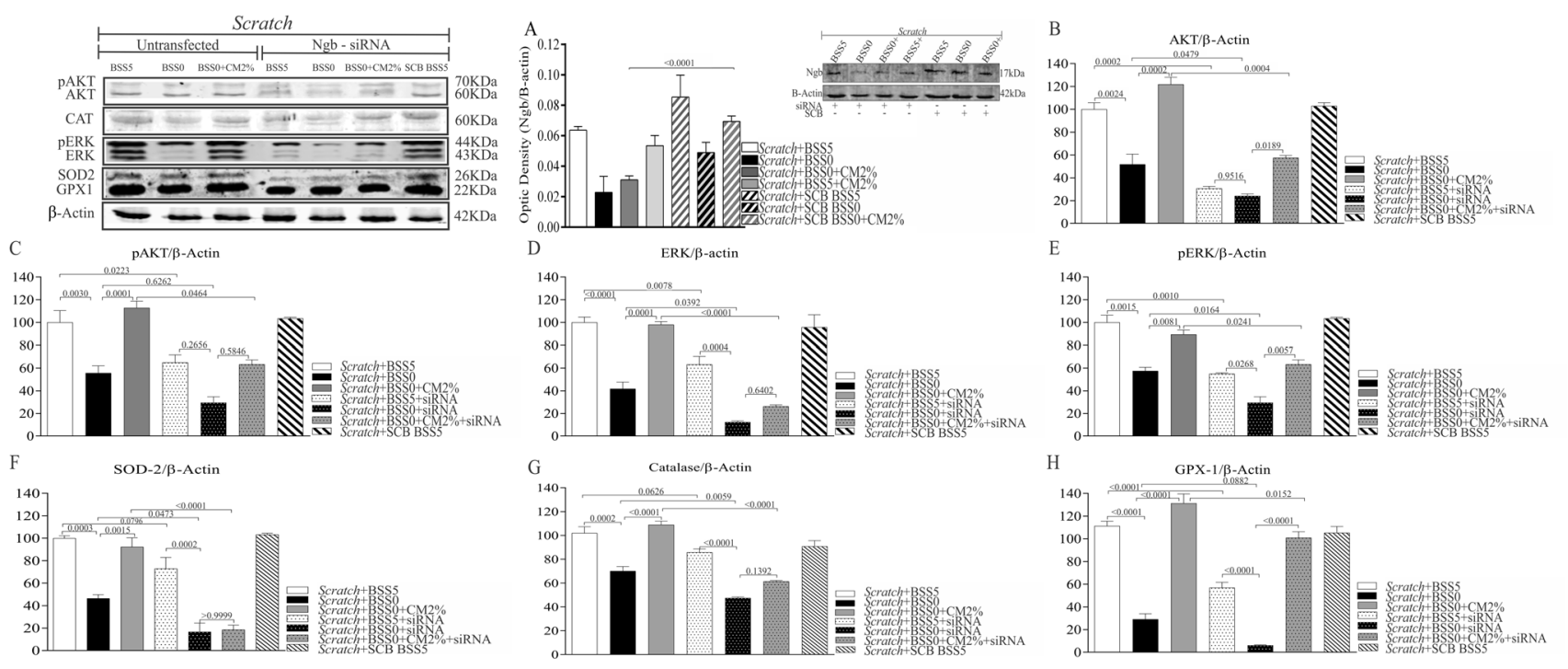

Publisher's Note Springer Nature remains neutral with regard to jurisdictional claims in published maps and institutional affiliations. 